\title{
Effectiveness of COVID-19 vaccine BNT162b2 at age 12-15 years with one dose
}

Oren Miron ${ }^{1}$, Rachel Wilf-Miron², Nadav Davidovitch ${ }^{1}$

1. Dept. of Health Systems Management, Ben-Gurion University, Israel

2. Faculty of Medicine, Tel Aviv University, Israel

Corresponding author: Oren Miron, Department of Health Systems Management, Ben-Gurion University, 1 David Ben-Gurion Blvd, 84105, Beer Sheva, Israel. Email: orenmir@post.bgu.ac.il. Phone: 972-8-6472448.

\section{Abstract:}

The clinical trial of BNT162b2 at age 12-15 years reported 100\% effectiveness in preventing COVID-19 after 7 days from dose-2, which is administrated at day 21 . We reanalyzed the data and found $100 \%$ effectiveness also after $14-20$ days from dose1 administration. This could relate to studies showing increased dose- 1 effectiveness at younger ages. Our findings support the possibility of vaccinating younger ages with the United Kingdom regimen, which delays dose-2 to day 84 to allow more dose-1 vaccinations.

\section{Introduction:}

The BNT162b2 clinical trial for age 16 year and above reported 95\% effectiveness after 7 days from dose-2, and a reanalysis of that data showed $93 \%$ effectiveness after 14-20 days from dose-1., ${ }^{1,2}$ The clinical trial of BNT162b2 at age 12-15 years reported $100 \%$ effectiveness after 7 days from dose-2, which lead us to reanalyze its data for the effectiveness after 14-20 days from dose- $1 .^{3}$ 


\section{Methods:}

We extracted data from the FDA report on the BNT162b2 clinical trial phase-3 for age 12-15 years, which had 2,260 participants divided between the BNT162b2 and the placebo groups. ${ }^{3}$ The extracted infection rate in the vaccinated group was divided by that of the placebo, and that value was subtracted from 1 to calculate the vaccine effectiveness. We calculated the effectiveness for days 14-20 from dose-1 administration.

\section{Results:}

The BNT162b2 group had 2 infections in its $1^{\text {st }}$ week, 1 infection in its $2^{\text {nd }}$ week, and 0 infections in its $3^{\text {rd }}$ week. The placebo group had 3 infections in its $1^{\text {st }}$ week, 4 infections in its $2^{\text {nd }}$ week and 5 infections in its $3^{\text {rd }}$ week (figure 1 ).

\section{Figure 1: Cumulative COVID-19 in BNT162b2 vs. placebo}

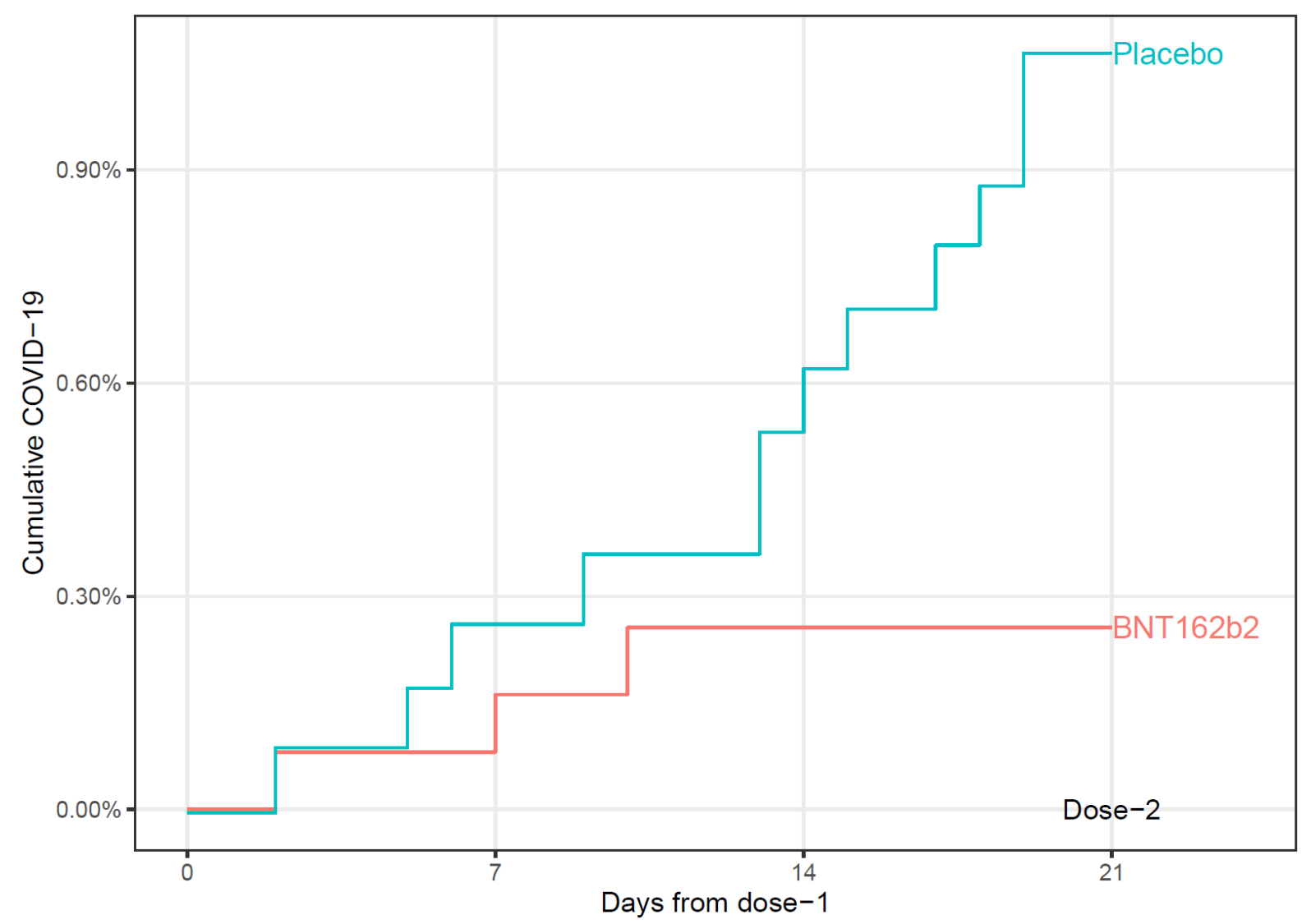


Legend: Y-axis indicates the cumulative COVID-19 infection rate. X-axis indicates the days from dose-1 administration. Blue indicates the BNT162b2 group while red indicates the placebo group.

The vaccine effectiveness in preventing COVID-19 was 50\% after $7-13$ days from dose-1, and $100 \%$ after $14-20$ days from dose-1 (figure 2 ).

\section{Figure 2: BNT162b2 effectiveness by days from dose-1 administration}

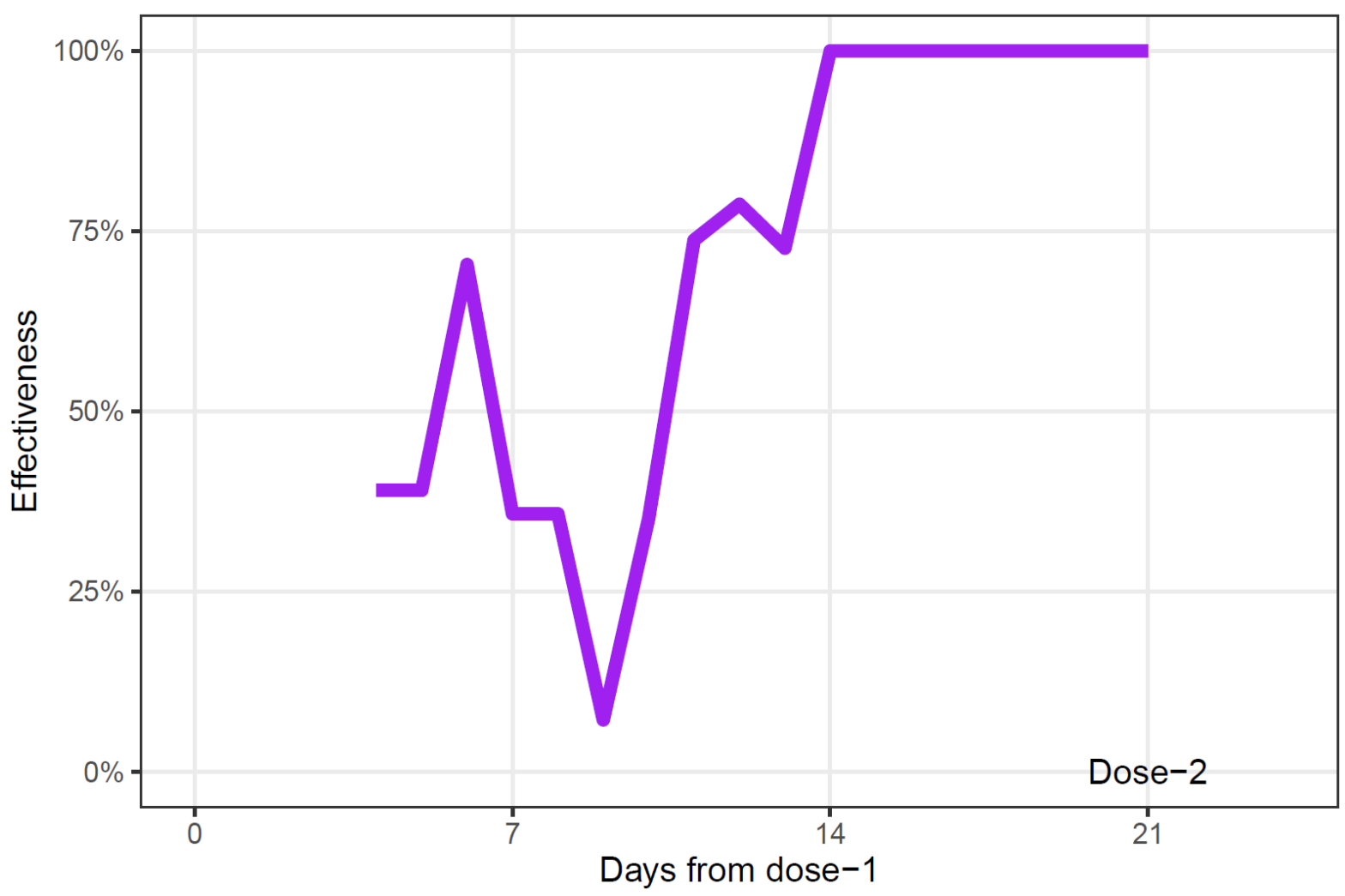

Legend: Y-axis indicates the effectiveness of BNT162b2 in preventing COVID-19 after a 7-day moving average. X-axis indicates the days from dose-1 administration.

\section{Discussion:}

Our reanalysis of data from the BNT162b2 clinical trial suggests that for age 12-15 years, effectiveness reached $100 \%$ in dose- 1 , which is the same as dose- 2 . This may relate to dose- 2 effectiveness reaching a ceiling effect at $100 \%$, and to studies 
finding higher dose-1 effectiveness at younger ages. ${ }^{4}$ Studies from the United

Kingdom, which delays dose-2 to vaccinate more people, have shown dose-1

effectiveness continues to rise for at least 60 days. ${ }^{5}$ Our finding suggest that such a strategy might be especially beneficial at younger ages since they have higher dose1 effectiveness.

\section{References:}

1 Polack FP, Thomas SJ, Kitchin N, et al. Safety and efficacy of the BNT162b2 mRNA Covid-19 vaccine. New England Journal of Medicine 2020.

2 Miron O, Wilf-Miron R, Davidovitch N. Effectiveness of COVID-19 Vaccines BNT162b2 and mRNA1273 by Days from Vaccination: A Reanalysis of Clinical Trial Data. Available at SSRN 37915602021.

3 Commissioner $\mathrm{O}$ of the. Pfizer-BioNTech COVID-19 Vaccine. FDA 2021; published online Nov 5. https://www.fda.gov/emergency-preparedness-and-response/coronavirus-disease-2019-covid19/pfizer-biontech-covid-19-vaccine (accessed May 12, 2021).

4 Yelin I, Katz R, Herzel E, et al. Associations of the BNT162b2 COVID-19 vaccine effectiveness with patient age and comorbidities. medRxiv 2021.

5 Hall VJ, Foulkes S, Saei A, et al. COVID-19 vaccine coverage in health-care workers in England and effectiveness of BNT162b2 mRNA vaccine against infection (SIREN): a prospective, multicentre, cohort study. The Lancet 2021; 0. DOI:10.1016/S0140-6736(21)00790-X. 